\title{
EFEITO DA ADIÇÃO DE CLORETO DE CÁLCIO NA ESTABILIDADE DA GORDURA EM SORVETES
}

FABIANO FREIRE COSTA*

JAIME VILELA DE RESENDE**

LUIZ RONALDO DE ABREU**

\begin{abstract}
Este trabalho objetivou estudar os efeitos da adição de cloreto de cálcio na coalescência dos glóbulos de gordura em sorvetes. Foram preparados sorvetes adicionados ou não de $0,025 \%$ de $\kappa$-carragena, $0,15 \%$ de emulsificante $(80 \%$ de mono e diglicerídeos e $20 \%$ de polissorbato 80 ) e cloreto de cálcio (0, 40 e $80 \mathrm{mM})$. Partículas coloidais presentes nas gotas do sorvete derretido foram analisadas e seus tamanhos e distribuição medidos, utilizando-se o equipamento Mastersizer 2000. De acordo com os resultados foi possível observar que não houve variação dos valores medidos nas amostras sem adição de cloreto de cálcio. Entretanto, quando se adicionou emulsificante ocorreu maior desestabilização dos glóbulos de gordura nas amostras de sorvetes com 40 e 80 mM de cloreto de cálcio e esses valores foram minimizados pela presença de $\kappa$-carragena. Os resultados podem ser explicados devido à capacidade de interação dos íons cálcio com os emulsificantes, estabilizantes e as proteínas do leite. Concluiu-se que a adição de cloreto de cálcio é extremamente danosa para a estabilidade de emulsões como sorvetes.
\end{abstract}

PALAVRAS-CHAVES: COALENCÊNCIA; GLÓBULOS DE GORDURA; SORVETE; CLORETO DE CÁLCIO.

* Doutor em Ciências dos Alimentos, Embrapa Gado de Leite, Juiz de Fora, MG (e-mail: fabianofreirecosta@ gmail.com).

** Professores, Laboratório de Microestrutura e Engenharia de Alimentos, Departamento de Ciências dos Alimentos, Universidade Federal de Lavras, Lavras, MG (e-mail: jvresende@ufla.br;|rabreu@ufla.br). 


\section{INTRODUÇÃo}

O sorvete é considerado como sistema complexo de partículas coloidais constituído de glóbulos de gordura, bolhas de ar e cristais de gelo dispersos numa fase não congelada (GOFF, 1992). O diâmetro das bolhas de ar e dos cristais de gelo variam de 20 a $50 \mu \mathrm{m}$, enquanto que os glóbulos de gordura apresentam diâmetros entre 0,1 a $1 \mu \mathrm{m}$ (CALDWELL, GOFF e STANLEY, 1992). A fase não congelada do sorvete é constituída de micelas de caseína suspensas em solução congelada e concentrada de açúcar, sais, proteínas solúveis do leite e polissacarídeos de elevada massa molecular (GOFF, 2003).

Durante o processo de preparo da mistura para sorvete, sob a ajuda de emulsificantes, os glóbulos de gordura e as proteínas do leite são adsorvidos uns aos outros formando emulsão coloidal. No processo de congelamento do sorvete, durante a batida da mistura, uma parte dos glóbulos de gordura que não se adsorveram nas proteínas do leite cristalizam-se formando estrutura de aparência sólida (GOFF, 1997). Outra parte desses glóbulos de gordura aglomera-se formando o fenômeno chamado de coalescência dos glóbulos de gordura. Esse fenômeno é comum e observado regularmente em sorvetes, porém seu excesso e o tamanho das partículas aglomeradas de glóbulos de gordura podem desencadear problemas estruturais como, por exemplo, o crescimento excessivo dos cristais de gelo (tamanho superior a $50 \mu \mathrm{m}$ de diâmetro), conferindo textura arenosa ao sorvete após a fabricação e principalmente durante a estocagem (COSTA et al., 2008). Equipamentos que medem a dispersão, a distribuição e o tamanho das partículas coloidais presentes em emulsões, como sorvetes, são comumente utilizados (GOFF et al., 2003) e os parâmetros obtidos indicam a existência de coalescência pelo tamanho e distribuição dos glóbulos de gordura.

Os emulsificantes e os estabilizantes polissacarídeos são ingredientes comumente usados em sorvetes no intuito de minimizar danos estruturais que ocorrem durante o processamento e a estocagem. Os emulsificantes são usados por facilitar a adsorção entre os glóbulos de gordura e as proteínas do leite, conferindo textura suave ao produto para agradar o paladar do consumidor (GOFF, 1999). Os emulsificantes podem ser divididos em dois grupos: os mono e diglicerídeos e os ésteres de sorbato. Os mono e diglicerídeos são derivados da reesterificação de gorduras animais hidrolisadas ou ácidos graxos extraídos de vegetais. A diferença entre os ésteres de sorbato e os monoglicerídeos está na estrutura química. Os ésteres de sorbato apresentam ácidos graxos, esterificados com moléculas de sorbitol, enquanto que os monoglicerídeos contém ácidos graxos esterificados com moléculas de glicerol. Para que os ésteres de sorbato se tornem hidrofílicos, durante sua produção, grupamentos polioxietileno são adicionados à estrutura carbônica. Desses ésteres de sorbato, o polisorbato 80 (monooleato polioxietileno de sorbato) é o mais comum emulsificante usado em sorvetes. Com o intuito de melhorar a ação dos emulsificantes, diversas indústrias alimentícias utilizam misturas de mono e diglicerídeos com o polisorbato 80 (MARSHALL et al., 2003). Em geral, os emulsificantes usados em sorvetes são constituídos basicamente de $80 \%$ de mono e diglicerídeos e $20 \%$ de polissorbato 80 .

Os estabilizantes polissacarídeos são substâncias hidrofílicas que dispersam em solução como coloides. A maioria desses produtos é classificada como gomas ou hidrocoloides, os quais podem ser definidos como qualquer polissacarídeo solúvel em água, extraído de vegetais terrestres, marinhos ou de microrganismos. Há, ainda, aqueles que podem sofrer modificações químicas para alcançar as características desejadas. São amplamente utilizados devido às características texturométricas, estruturais e funcionais, proporcionando estabilidade a emulsões, suspensões e espumas, e pelas suas propriedades espessantes em geral (FENNEMA, 1993). Em solução, os estabilizantes aumentam a viscosidade e formam geis, devido à sua capacidade de retenção de grande quantidade de água (água livre), resultante da atividade mútua desses compostos que formam ligações de hidrogênio entre si. A capacidade de retenção da água livre torna esse produto ingrediente indispensável para sorvetes por diminuir a quantidade de água que cristaliza durante o congelamento, aumentando a viscosidade e dessa forma evitando que os cristais de gelo se 
fundam causando o defeito de arenosidade (FENNEMA, 1991). A к-carragena está incluída como estabilizante para sorvetes (MARSHALL, GOFF e HARTEL, 2003). Esse biopolímero em solução apresenta-se negativamente carregado devido à presença de grupamento sulfatado em cada dissacarídeo galactana (REES et al., 1969). Sua principal função é limitar a separação de fases entre os demais polissacarídeos adicionados ao sorvete e as proteínas do leite (SCHORSCH, JONES e NORTON, 2000: SPAGNUOLO et al., 2005; VEGA, DALGLEISH e GOFF, 2005).

A adição de minerais e vitaminas aos alimentos, praticada pelas indústrias alimentícias, visa chamar a atenção do consumidor e dessa forma agregar valor aos produtos enriquecidos e/ou fortificados (WEAVER, 1998). A existência de relação entre a baixa ingestão de cálcio com doenças como osteoporose (USDA, 2005) vêm despertando o interesse das indústrias alimentícias em fortificar e/ou enriquecer alimentos com esse mineral. Entretanto, diversos trabalhos (WILLIANS, D'ATH e AUGUSTIN, 2005; COSTA et al., 2008, DAUPHAS et al., 2008) têm demonstrado que a adição de cálcio na forma natural (iônica) é extremamente danosa à estrutura e qualidade dos alimentos industrializados devido sua capacidade de interação com carboidratos, lipídeos e proteínas (WEAVER e HEANEY, 2006).

Este trabalho objetivou investigar o efeito de diferentes níveis de cloreto de cálcio em sorvetes adicionados ou não de $\kappa$-carragena e emulsificante por meio da medida da coalescência das partículas de gordura.

\section{MATERIAL E MÉTODOS}

\subsection{PREPARO DAS AMOSTRAS}

O preparado para sorvetes consistiu de mistura contendo 10\% de gordura (manteiga doce, Gay Lea Foods, Guelph, ON, Canadá), 11\% de sólidos não gordurosos (leite em pó, Gay Lea Foods, Guelph, ON, Canadá), 12\% de sacarose (Redpath Sugar, Toronto, ON, Canadá), 4\% de xarope de milho (Casco Inc., Toronto, ON, Canadá), 0 ou $0,025 \%$ de estabilizante ( $\kappa$-carragena; Germantown Canadá Inc., Scarborough, ON), 0 ou 0,15\% de emulsificante (POLMO: $80 \%$ de mono e diglicerídeos e $20 \%$ de polissorbato 80; Danisco, Aarhus, Dinamarca), cloreto de cálcio (Fisher, Toronto, Canadá) e água para o balanço total de $100 \%$. Os diferentes tratamentos adicionados ou não de к-carragena, POLMO e cloreto de cálcio (nas proporções de 0,40 e $80 \mathrm{mM}$ de $\mathrm{Ca}^{2+}$ ) foram preparados e encaminhados ao homogeneizador (17.5/3.5 Mpa, APV Gaulin, Everett, MA), pasteurizados $\left(75^{\circ} \mathrm{C} / 15\right.$ minutos), maturados $\left(24\right.$ horas $/ 4^{\circ} \mathrm{C}$ ), batidos (Taylor Batch Freezer, model 104 , Rockton, IL) à temperatura de $-5^{\circ} \mathrm{C}$ e armazenados por 48 horas à temperatura de $-35^{\circ} \mathrm{C}$. Todas as amostras foram preparadas em triplicata.

\subsection{MEDIDA DA COALESCÊNCIA DAS PARTÍCULAS}

Partículas coloidais presentes nas gotas de sorvete derretido dos diferentes tratamentos foram caracterizadas de acordo com a distribuição, tamanho e área superficial de contato pela técnica de difração de luz estática, utilizando-se o equipamento Mastersizer 2000 (Malvern Instruments, Malvern, UK). A razão entre o índice de refração dos glóbulos de gordura $(1,456)$ e o índice de refração da fase contínua $(1,330)$ foi de 1,095 . As médias do tamanho dos glóbulos de gordura foram obtidas como diâmetro médio de De Brouckere $\left(d_{[4,3]}=\sum n_{i} d_{i}^{4} / \sum n_{i} d_{i}^{3}\right)$ ou diâmetro médio de Sauter $\left(\mathrm{d}_{[3,2]}=\Sigma n_{i} \mathrm{~d}_{\mathrm{i}}{ }^{3} / \Sigma n_{\mathrm{i}} \mathrm{d}_{\mathrm{i}}{ }^{2}\right.$, em que $\mathrm{n}_{\mathrm{i}}=$ o número de glóbulos de gordura com diâmetro $\left.\mathrm{d}_{\mathrm{i}}\right)$. Valores para área específica de contato, $\mathrm{m}^{2} \mathrm{~g}^{-1}$, foram também medidos (COSTA et al., 2008).

\subsection{ANÁLISE ESTATÍSTICA}

Adotou-se delineamento experimental inteiramente casualizado (DIC), em esquema fatorial 
$2 \times 2 \times 3 \times 3$ (adicionados ou não de $\kappa$-carragena, emulsificante e cloreto de cálcio, nas proporções de 0,40 e $80 \mathrm{mM}$ de $\mathrm{Ca}^{2+}$ ). Todos os experimentos foram realizados em triplicata. Aplicou-se o teste de Tukey para determinar a significância das diferenças entre os tratamentos. As análises foram realizadas com auxílio do programa SISVAR 4.3 (FERREIRA, 1999), utilizando-se planilha eletrônica Excel.

\section{RESULTADOS E DISCUSSÃO}

As Tabelas 1, 2, 3 e 4 mostram os resultados quantitativos que caracterizam a desestabilização dos glóbulos de gordura nas amostras de sorvetes com ou sem estabilizante ( $\kappa$-carragena), emulsificante ( $80 \%$ de mono e diglicerídeos e $20 \%$ de polissorbato 80 ) e cloreto de cálcio adicionado à mistura básica para sorvetes em diferentes concentrações $(0,40$ e $80 \mathrm{mM})$. As Tabelas apresentam, respectivamente, os valores médios obtidos para diâmetro ponderado pelo volume $\left(\mathrm{d}_{[4,3]}\right)$, diâmetros ponderados pela área superficial $\left(\mathrm{d}_{[3,2]}\right)$, área superficial específica e a porcentagem das partículas com diâmetro superior a $3 \mu \mathrm{m}$.

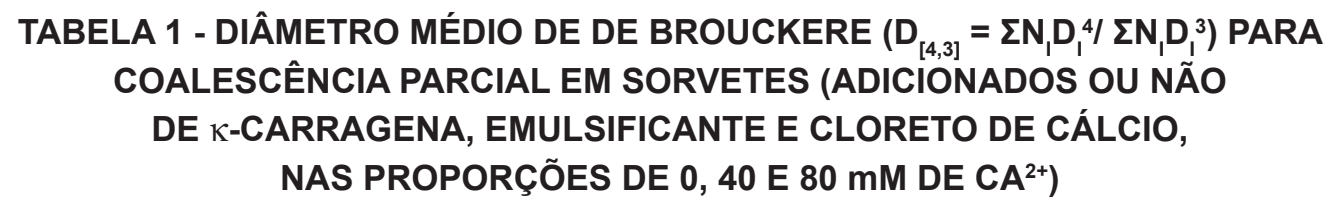

\begin{tabular}{cccc}
\hline \multirow{2}{*}{$\begin{array}{c}\text { Cálcio } \\
(\mathrm{Mm})\end{array}$} & $\begin{array}{c}\text { } \text {-Carragena } \\
(0,025 \%)\end{array}$ & \multicolumn{2}{c}{ Emulsificante $(0,15 \%)$} \\
\cline { 3 - 4 } & Com & $3,97^{\mathrm{a} 1}$ & Sem \\
\hline 0 & Sem & $6,10^{\mathrm{b} 1}$ & $1,44^{\mathrm{a} 2}$ \\
0 & Com & $6,20^{\mathrm{a} 1}$ & $0,86^{\mathrm{a} 2}$ \\
\hline 40 & Sem & $2,81^{\mathrm{b} 1}$ & $1,07^{\mathrm{a} 2}$ \\
40 & Com & $6,27^{\mathrm{a} 1}$ & $1,51^{\mathrm{a} 2}$ \\
80 & Sem & $4,47^{\mathrm{b} 1}$ & $2,77^{\mathrm{a} 2}$ \\
80 & & $2,91^{\mathrm{a} 2}$ \\
\hline
\end{tabular}

Letras diferentes na coluna e números diferentes na linha são estatisticamente significativos pelo teste de Tukey $(P<0,05)$.
TABELA 2 - DIÂMETRO MÉDIO DE SAUTER $\left(D_{[3,2]}=\Sigma N_{1} D_{1}^{3} / \Sigma N_{1} D_{1}^{2}\right.$, EM QUE $N_{1}=0$ NÚMERO DE GLÓBULOS DE GORDURA COM DIÂMETRO D, PARA A COALESCÊNCIA PARCIAL
EM SORVETES (ADICIONADOS OU NÃO DE K-CARRAGENA, EMULSIFICANTE E CLORETO DE CÁLCIO, NAS PROPORÇÕES DE 0, 40 E 80 mM DE CA ${ }^{2+}$ )

\begin{tabular}{|c|c|c|c|}
\hline \multirow{2}{*}{$\begin{array}{l}\text { Cálcio } \\
(\mathrm{Mm})\end{array}$} & \multirow{2}{*}{$\begin{array}{c}\kappa \text {-Carragena } \\
(0,025 \%)\end{array}$} & \multicolumn{2}{|c|}{ Emulsificante $(0,15 \%)$} \\
\hline & & Com & Sem \\
\hline 0 & Com & $0,41^{a 1}$ & $0,35^{a 1}$ \\
\hline 0 & Sem & $0,56^{\mathrm{b} 1}$ & $0,27^{\mathrm{a} 2}$ \\
\hline $40^{-}$ & Com & $0,81^{\mathrm{a} 1}$ & $0,31^{\mathrm{a} 2}$ \\
\hline 40 & Sem & $0,37^{\mathrm{b} 1}$ & $0,35^{\mathrm{a} 1}$ \\
\hline 80 & Com & $0,70^{\mathrm{a} 1}$ & $0,38^{\mathrm{a} 2}$ \\
\hline 80 & Sem & $0,60^{a 1}$ & $0,37^{\mathrm{a} 2}$ \\
\hline
\end{tabular}

Letras diferentes na coluna e números diferentes na linha são estatísticamente significativos pelo teste de Tukey $(P<0,05)$. 


\section{TABELA 3 - ÁREA SUPERFICIAL ESPECÍFICA DAS PARTÍCULAS COLOIDAIS EM SORVETES (ADICIONADOS OU NÃO DE K-CARRAGENA, EMULSIFICANTE E CLORETO DE CÁLCIO, NAS PROPORÇÕES DE 0, 40 E 80 mM DE CA ${ }^{2+}$ )}

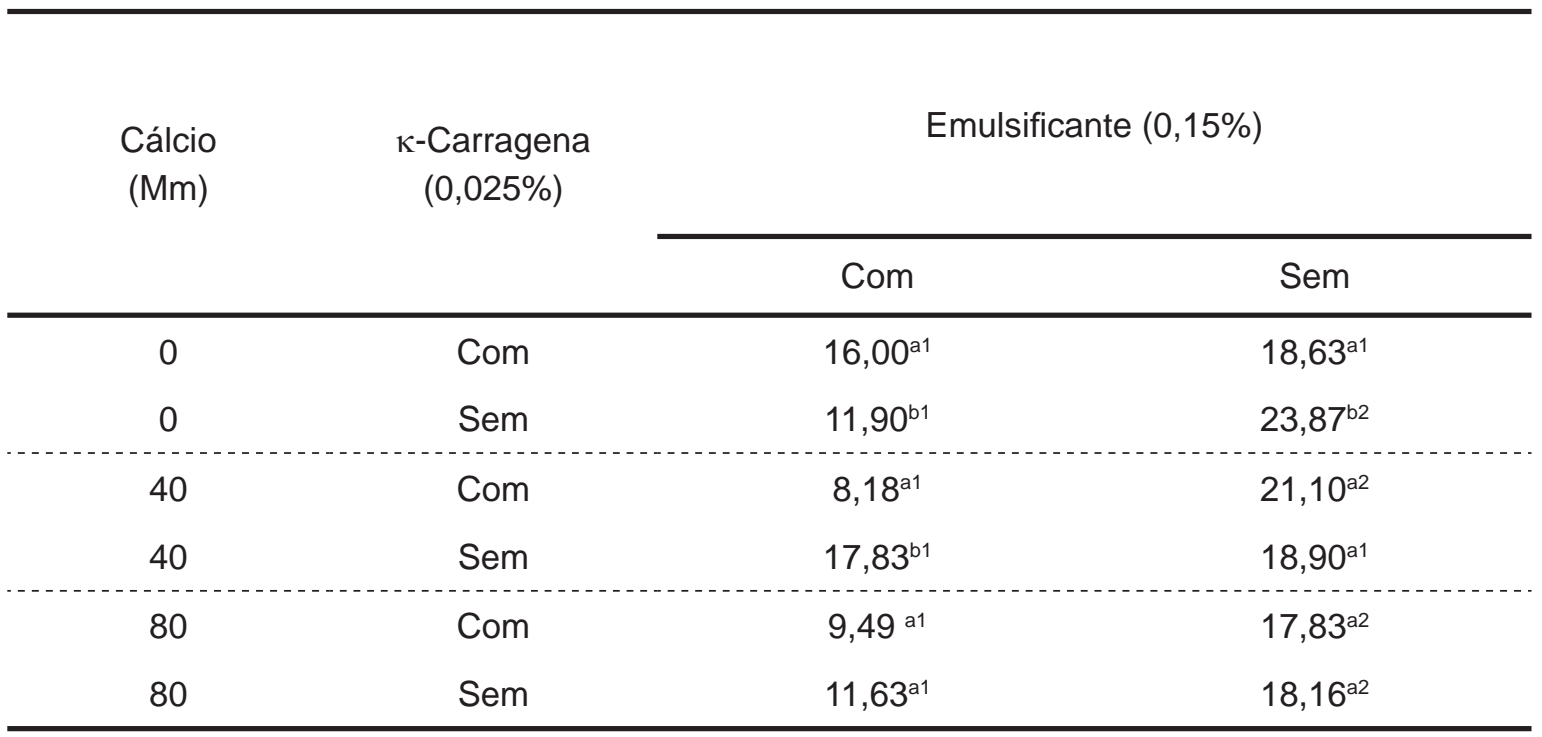

Letras diferentes na coluna e números diferentes na linha são estatísticamente significativos pelo teste de Tukey $(P<0,05)$.

\section{TABELA 4 - DIÂMETRO MÉDIO DAS PARTÍCULAS ACIMA DE $3 \mu \mathrm{M}$ NO SORVETE ADICIONADO DE 40 mM DE CLORETO DE CÁLCIO}

\begin{tabular}{ll}
\hline Com & $30,11^{\mathrm{a}}$ \\
Sem & $17,26^{\mathrm{b}}$ \\
\hline
\end{tabular}

Letras diferentes na coluna são estatísticamente significativos pelo teste de Tukey $(P<0,05)$.

De acordo com os resultados (Tabelas 1, 2 e 3) foi possível observar que sem emulsificante não houve variação estatisticamente significativa $(P>0,05)$ para ausência ou presença de $\kappa$-carragena e cloreto de cálcio nas diferentes concentrações. Esses baixos valores para a medida de desestabilização dos glóbulos de gordura estão de acordo com a descrição efetuada por Goff (2003). No entanto, quando se adicionou emulsificante $(0,15 \%)$ observou-se comportamento estatisticamente significativo $(P<0,05)$ para a presença ou ausência de $\kappa$-carragena $(0,025 \%)$ e cloreto de cálcio (40 e 80 mM). Quando não se adicionou cloreto de cálcio (Tabelas 1, 2 e 3) houve indicativo maior de coalescência (maior valor para diâmetro ponderado pelo volume $\left(\mathrm{d}_{[4,3]}\right)$ e diâmetros ponderados pela área superficial $\left(\mathrm{d}_{[3,2]}\right)$, e baixo valor para área superficial específica) para as amostras sem adição de $\kappa$-carragena. Na presença de $\kappa$-carragena, o indicativo de coalescência diminui (Tabelas 1, 2 e 3) mostrando menores valores para o diâmetro ponderado pelo volume $\left(\mathrm{d}_{[4,3]}\right)$, diâmetro ponderado pela área superficial $\left(\mathrm{d}_{[3,2]}\right)$ e maior valor para área superficial específica. Quando se adicionou cloreto de cálcio (40 e $80 \mathrm{mM}$ ) ocorreu inversão dos resultados. Os indicativos de coalescência aumentaram para presença de $\kappa$-carragena (Tabelas 1, 2 e 3), sendo observados maiores valores para diâmetro ponderado pelo volume $\left(\mathrm{d}_{[4,3]}\right)$ e diâmetros ponderados 
pela área superficial $\left(\mathrm{d}_{[3,2]}\right)$ e menores valores para área superficial específica. Os resultados para diâmetros de partículas superiores a $3 \mu \mathrm{m}$ (Tabela 4) mostraram que na presença de cloreto de cálcio $(40 \mathrm{mM}$ ou $80 \mathrm{mM})$ existe variação estatisticamente significativa $(P<0,05)$ para a adição ou não de emulsificante $(0,15 \%)$. De acordo com a Tabela 4 , cerca de $30,11 \%$ das partículas de gordura presentes nas amostras de sorvetes adicionados de emulsificante ficaram com diâmetros superiores a $3 \mu \mathrm{m}$, ao passo que $17,26 \%$ das partículas de sorvetes sem adição de emulsificante ficaram abaixo desse valor.

Os efeitos da adição de cloreto de cálcio e suas interações em emulsões lácteas, como sorvetes, foram observados nos estudos realizados por Dauphas et al. (2008) e Costa et al. (2008). Especificamente em sorvetes, Costa et al. (2008) verificaram que a adição de cloreto de cálcio impactou negativamente na estrutura da emulsão e esse efeito foi acentuado pela presença de $\kappa$-carragena. De acordo com os resultados obtidos anteriormente, quando se adicionou cloreto de cálcio ao sorvete constatou-se considerável coalescência dos glóbulos de gordura, ocasionando desestabilização da emulsão e consequentemente crescimento dos cristais de gelo a diâmetros superiores a $50 \mu \mathrm{m}$.

Os íons cálcio também influenciaram drasticamente os sorvetes adicionados ou não de emulsificante. Nos resultados obtidos para coalescência da gordura foi observado que os íons cálcio interagiram com os emulsificantes, nesse caso constituídos basicamente de $80 \%$ de mono e diglicerídeos e $20 \%$ de polissorbato 80 . Os ésteres de sorbato contém ácidos graxos esterificados com moléculas de sorbitol, enquanto os monoglicerídeos apresentam ácidos graxos esterificados com moléculas de glicerol. Para que os ésteres de sorbato se tornem hidrofílicos grupamentos polioxietileno são adicionados à estrutura carbônica, os quais se apresentam como negativos em solução, o que facilita a interação com íons cálcio. Tais interações inativam os emulsificantes que são usados com o objetivo de melhorar as interações entre os glóbulos de gordura e as proteínas presentes no sorvete, desestabilizando a estrutura e, consequentemente, aumentando os raios de crescimento dos cristais de gelo (FENNEMA, 1993).

As interações entre a א-carragena e as micelas de caseína e a inativação dos emulsificantes causaram, provavelmente, a grande redução da estabilidade dos glóbulos de gordura devido à diminuição da superfície de adsorção entre as proteínas.

\section{CONCLUSÃO}

A fortificação de cálcio pela adição de cloreto de cálcio em formulações convencionais de sorvete, usando-se sólidos não gordurosos do leite, induz a impactos negativos nas propriedades físicas, estruturais e de qualidade dos sorvetes, cujos efeitos foram acentuados pela presença de $\kappa$-carragena. Cerca de $30,11 \%$ das partículas de gordura presentes em amostras de sorvetes adicionadas de emulsificante evidenciaram diâmetros superiores a $3 \mu \mathrm{m}$, ao passo que $17,26 \%$ das partículas de sorvetes sem adição de emulsificante ficaram abaixo desse valor. Isto explica os efeitos observados e principalmente as interações existentes entre os íons cálcio e os emulsificantes.

\section{ABSTRACT}

\section{FAT DESTABILIZATION IN ICE CREAM BY CALCIUM CHLORIDE ADDITION}

The objective of this work was to investigate the effect of calcium chloride addition over the coalescence of fat globules in ice cream. Were prepared ice creams with and without $\kappa$-carrageenan $0.025 \%, 0.15 \%$ emulsifier ( $80 \%$ of mono- and di-glycerides plus $20 \%$ polysorbate 80$)$ and calcium chloride content $(0,40$ and $80 \mathrm{mM}$ ). Colloidal particles present in drops of melted ice cream were analyzed and its size and distribution determined using the Mastersizer 2000 equipment. According to the results it was possible to verify that 
in the samples without calcium chloride measurements did not vary. However the addition of emulsifier led to an increase of destabilization of the fat globules, as observed in the samples with 40 and $80 \mathrm{mM}$ of calcium chloride and those values were minimized by the presence of k-carrageenan. It was concluded that the addition of calcium chloride is extremely detrimental for the stability of emulsions such as ice cream.

KEY-WORDS: COALESCENCE; ICE CREAM; FAT GLOBULES; CALCIM CHLORIDE.

\section{REFERÊNCIAS}

1 CALDWELL, K. B.; GOFF, H. D.; STANLEY, D. W. A low-temperature scanning electron microscopy study of ice cream. I. Techniques and general microstructure. Food Structure, Chicago, v.11, n.1, p.1-9, 1992.

2 COSTA, F. F.; RESENDE. J. V.; ABREU, L. R.; GOFF, H. D. Effect of calcium chloride addition on ice cream structure and quality. Journal of Dairy Science, Champaign, v.91, n.6, p.2165-2174, June 2008.

3 COSTA, F. F. Efeitos da fortificação com cálcio na estrutura de sorvetes. 2008. 57 p. Tese (Doutorado em Ciências dos Alimentos) - Universidade Federal de Lavras, Lavras, 2008.

4 DAUPHAS, S.; AMESTOY, A.; LLAMAS, G.; ANTON, M.; RIAUBLANC, A. Modification of the interactions between $\beta$-casein stabilized oil droplets with calcium addition and temperature changing. Food Hydrocolloids, Oxford, v.22, n.2, p.231-238, Mar. 2008.

5 FENNEMA, O. R. Instability of nonequilibrium states of water in frozen foods. Ciência e Tecnologia de Alimentos, Campinas, v.11, n.2, p.152-169, maio/ago. 1991.

6 FENNEMA, O. R. Química de los alimentos. 2. ed. Zaragoza: Acribia, 1993.

7 FERREIRA, D. F. SISVAR - sistema de análise de variância para dados balanceados: programa de análises estatísticas e planejamento de experimentos. Versão 4.3. Lavras: UFLA, 1999.

8 GOFF, H. D. Low-temperature stability and the glassy state in frozen foods. Food Research International, Ontario, v.25, n.4, p.317-325, 1992.

9 GOFF, H. D. Colloidal aspects of ice cream. International Dairy Journal, Oxford, v.7, n.6-7, p.363-373, June/July 1997.

10 GOFF, H. D.; VERESPEJ, E.; SMITH, A.K. A study of fat and air structures in ice cream. International Dairy Journal, Oxford, v.9, n.11, p. 817-829, 1999.

11 GOFF, H. D. Ice cream. In: FOX, P.F.; MCSWEENEY, P.L.H. (Ed.). Advanced dairy chemistry: proteins. $3^{\text {rd }}$ ed. New York: Kluwer, 2003. p.1063-1085.

12 MARSHALL, R. T.; GOFF, H.D.; HARTEL, R.W. Ice cream. $6^{\text {th }}$ ed. New York: Kluwer Academic, 2003.

13 REES, D. A. I.; STEELE, W.; WILLIAMSON, F. B. Conformational analysis of polysaccharides. III. The relation between stereochemistry and properties of some polysaccharides sulfates (I). Journal of Polymer Science; Part C, New York, v.28, p.261-276, 1969.

14 SCHORSCH, C.; JONES, M. G.; NORTON, I. T. Phase behaviour of pure micellar casein/ k-carrageenan systems in milk salt ultrafiltrate. Food Hydrocolloids, Oxford, v.14, n.4, p.347-358, July 2000.

15 SPAGNUOLO, P. A.; DALGLEISH, D. G.; GOFF, H. D.; MORRIS, E. R. Kappa-carrageenan interactions in systems containing casein micelles and polysaccharide stabilizers. Food Hydrocolloids, Oxford, v.19, n.3, p.371-377, May 2005.

16 UNITED STATES DEPARTMENT OF AGRICULTURE. Continuing survey of food intakes by individuals, 2000-2002. Washington, DC: National Health and Nutrition Examination Survey, 2005.

17 EGA, C.; DALGLEISH, D. G.; GOFF, H. D. Effect of k-carrageenan addition to dairy emulsions 
containing sodium caseinate and locust bean. Food Hydrocolloids, Oxford, v.19, n.2, p.187-195, Mar. 2005.

18 WEAVER, C. M. Calcium in food fortification strategies. International Dairy Journal, v.8, n.5-6, p. 443-449, May/June 1998.

19 WEAVER, C. M.; HEANEY, R. P. Calcium in human health. Totowa: Humana, 2006. p. 137.

20 WILLIANS, R. P. W.; D'ATH, L.; AUGUSTIN, M. A. Production of calcium-fortified milk powders using soluble calcium salts. Lait, Paris, v.85, n.4-5, p.369-381, July/Oct. 2005. 